\title{
The Poppy Humanoid Robot: Leg Design for Biped Locomotion
}

\author{
Matthieu Lapeyre ${ }^{1}$, Pierre Rouanet ${ }^{1}$ and Pierre-Yves Oudeyer ${ }^{1}$
}

\begin{abstract}
We introduce a novel humanoid robotic platform designed to jointly address three central goals of humanoid robotics: 1) study the role of morphology in biped locomotion; 2) study full-body compliant physical human-robot interaction; 3) be robust while easy and fast to duplicate to facilitate experimentation. The taken approach relies on functional modeling of certain aspects of human morphology, optimizing materials and geometry, as well as on the use of $3 \mathrm{D}$ printing techniques. In this article, we focus on the presentation of the design of specific morphological parts related to biped locomotion: the hip, the thigh, the limb mesh and the knee. We present initial experiments showing properties of the robot when walking with the physical guidance of a human.
\end{abstract}

\section{INTRODUCTION}

Research in humanoid robotics has been thriving in the recent years [13] [16], both due to the predicted relevance of humanoid robots for personal and assistive robotics [37], and due to fundamental scientific questions they raise with regards to biped locomotion and full-body physical interaction with the environment. Indeed, biped robots need to be able to move robustly and efficiently in human environments, which include the ability to keep stability when unpredictable physical contact with humans happens. At the same time, these robots need to be capable of rich and safe social and physical interaction with humans, and to adapt to the behavior and preferences of each particular user.

We should not only try to solve these challenges through artificial cognitive intelligence but also through body intelligence [29]. On one hand, a way to permit robots to adapt their behaviors to unknown environments is to provide them with control algorithms which can be updated with learning algorithms based on social guidance [6], or on autonomous self-exploration [4][17]. On the other hand, a part of the computation needed for such adaptation could also be done through the intrinsic mechanics and electronics of the robot, thus providing effective and hyper-responsive reactions while simplifying the algorithms of the different behaviors. This role of morphology has been called morphological computation [30], as the body of the robot becomes a form of information processing structure, capable of supporting a part of the computation necessary to achieve sensorimotor tasks to simplify or make it more robust to external disturbances [30][32]. The actions or reactions of the physical body also have the advantage of being direct without latency due to a controller, as opposed to CPU computed reactions which often require high-cost hardware in order to respond fast enough and reduce modeling errors.

The work presented in this article takes place within a research program exploring which mechanisms can allow humanoid robots to acquire sensorimotor and social skills in a life-long manner, and through self-exploration and social interaction with non-technical users [40][18][27][25][26]. A central vision of this research program, deeply inspired by infant learning and development, is that life-long skill learning in the real world can only effectively happen if statistical inference is guided by strong constraints, in particular

\footnotetext{
*This research was partially funded by ERC Starting Grant EXPLORERS 240007.

1 INRIA Flowers Team, Bordeaux, France matthieu.lapeyre, pierre.rouanet, pierre-yves.oudeyer at inria.fr
}

related to the physics of the body (their material, their geometry and the evolution of this geometry as the body grows [5][3][17]) and to the social environment [6][1]. Indeed, typical humanoid bodies are high-dimensional, which is extremely challenging for acquiring sensorimotor controller. Body intelligence and social guidance have been argued to facilitate and considerably guide the learning and development of sensorimotor skills in these complex spaces [26]. Within this context, we present in this article the humanoid robot Poppy, which elaboration was done to address the following design goals:

Morphology and biped locomotion: Studying the impact of morphology over the control and/or learning of skills requires the possibility to implement and experiment novel morphologies. Two particularly interesting skills are biped locomotion and robustness to physical interaction with humans. Poppy uses the bio-inspired trunk developed for the Acroban humanoid robot [19], useful for both these skills. In addition, it also includes a novel hip and thigh physical design which is presented and studied in this article with regards to its impact on locomotion and balance control, as well as optimized design of knees and compliant feet. The geometry of limbs has been optimized to minimize weight thanks to a mesh structure and optimal use of motors, which in turn have allowed the decrease of motor power and weight, as well as energy consumption, while reinforcing safety (see below). Such morphological designs were made possible with the use of 3D printing techniques.

Social and physical human-robot interaction: Most often, robots designed to study the role of morphology in biped locomotion do not afford rich social and physical interaction with humans: with a minimal torso and no head [8][24]. Poppy was designed to afford such full-body physical interaction (we will illustrate this with the possibility to guide him physically in biped locomotion), as well as to afford social interaction, with a head and gestural apparatus that can be programmed for communicative or affective expression.

Full-body compliance: Important aspects of adaptation to physical obstacles or to humans require humanoid robots to be fullbody compliant. This includes both the ability to absorb external shocks due to the passive compliance of the mechanical structure (bendable materials and springs), but also the ability to actively and dynamically control the compliance of motors, which may be either controlled in position with compliance, or directly in torque (thanks to the use of adequate recent servomotor technologies).

Robustness and Safety: The above mentioned research endeavor requires that heavy and long real-world experimentation be conducted with the robot. This implies that the robot should be robust and safe. It should be able to sustain experiments and fall down without easily breaking. At the same time, one should ensure that physical interaction with the robot is safe for humans. The approach taken is again based on morphological design, where the combination of lightweight design, compliance, and robust materials is used.

Breakable, repairable: Even if breaking should be made unusual, real-world experimentation should be expected to break the 
robot at regular intervals. This should not become a problem for conducting research. Breaking should not be costly and the robot should be easily repairable. This is achieved thanks to 3D printing techniques, affordable off-the-shelf components, and optimized mounting design.

Precision, stationarity: Experiments should be repeatable, implying that the robot properties should be stationary.

Transportable outside the lab: To allow for experiments in natural environments, possibly involving interaction with non-technical humans, the robot should be transportable outside the laboratory.

Easy and fast to duplicate: Such a reuse of the robotic platform requires that it is easy and fast to duplicate. The approach taken is to only use off-the-shelf components (motors and electronics) and limbs which can be printed with regular $3 \mathrm{D}$ printing services. The Poppy humanoid platform takes two days to assemble by one user, and was already reproduced twice, including by another laboratory 1

Affordable: A mid-term goal of this project is to open the hardware and software platform to the academic community (under an open-source mode), to allow other research laboratories to use it as an experimental platform. A key aspect for such an open dissemination is to keep the cost of the robot relatively low. The overall materials needed to build a Poppy robot cost around 7500 euros including 4700 euros for actuators, 1600 euros for of the shell mechanical and electronic components and 1200 euros for 3D printed mechanical parts.

The Poppy platform presented in this article was designed to target these design goals within the context of biped locomotion. We focus here on the presentation of the design of specific morphological parts: the hip, the thigh, the limb mesh and the knee.

\section{RELATED WORK}

Regarding the role of morphology in biped locomotion, one of the first famous example concerns the work of Tad MacGeer on passive dynamic walkers [21]. Thanks to the understanding of the intrinsic dynamics of its structure, McGeer has managed to create a $2 \mathrm{D}$ biped robot capable of producing several steps without any controller or motor. The only control of this robot is due to the interaction between the intrinsic inertia of the structure and gravity. This work has been pursued by those of Collins [8] and Tedrake [38] who perfected the concept to make 3D walkers possible and over longer distances. The structure of its robot has its own dynamic that allow it to self-stabilize and maintain a walking motion.

The concept of morphological computation has also been associated to the principle of "ecological balance", as outlined by Pfeifer et al. [31], which states that there is a balance or task distribution between morphology, materials, control, and interaction with the environment. For example, morphological computation has been shown to be necessary in order to achieve human-like biped locomotion [20] and the coupling of adequate morphologies with central-pattern generators has been shown to generate robust locomotor behavior [15][36].

It has also been shown that the compliance of the body explains the dynamics of walking and running [10] and several biped robots such as Athlete Robot [24] or BioBiped1 [33] were designed using compliant actuator or elastic material. These robots showed interesting hopping and running behavior while using less power actuator than common humanoid robot such as Asimo or HRP-2.

Among all robots designed to explore morphological computation and compliant body only few allow to explore physical interaction

\footnotetext{
${ }^{1}$ Laboratoire de la Perception et de l'Action (J. Droulez), Collège de France, Paris, France
}

such as Kenshiro [2] or Acroban which the compliant structure of its vertebral column and legs was shown to permit a self-organized physical human-robot interface allowing non-expert users to lead the robot by the hand [19][28].

The morphological properties of these robotic platforms are especially interesting but unfortunately they are difficult and expensive to reproduce by other research laboratories.

Other current research platforms are easily accessible and easy to use such as Nao [11], Darwin Op [12], Nimbro Op [34] or iCub [22]. Yet, they provide a "traditional" morphology (e.g. limited compliance, rigid torso, big feet, over actuated) which can not be easily modified. It makes them unadapted to study the impact of the morphology on biped locomotion and human physical interaction.

Each of these platforms provides key features for robotics but none of them regroups all the ones needed to explore both biped locomotion and physical interaction.

The following table (Fig[1) summarizes the main goals of the Poppy platform as described in $[$ and gives assessment with respect to these goals to a selection of relevant robotic platforms.

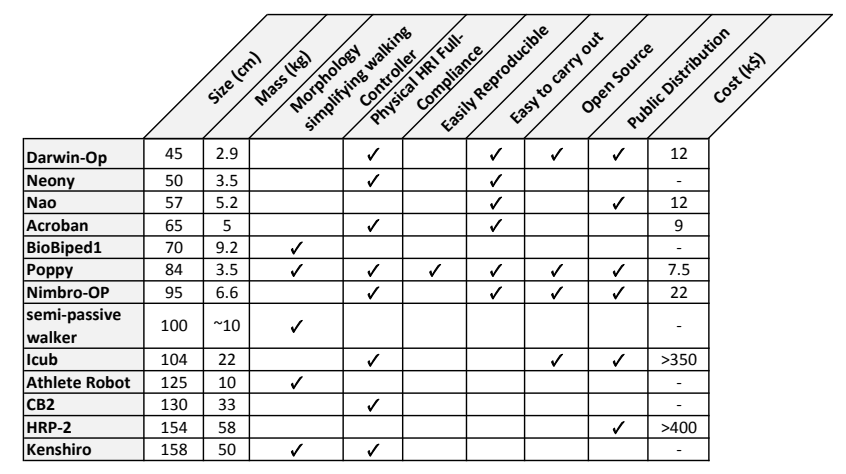

Fig. 1: Comparison of different robots on main goals addressed by the Poppy platform

\section{The Poppy HumanoId Platform}

Poppy (Fig. 2) is a $84 \mathrm{~cm}$ high humanoid robot which weights $3.5 \mathrm{~kg}$. It has a sensorimotor space consisting of 25 motorized joints using Robotis Dynamixel servomotors (MX-28 and AX-12). These servo-motors give access to a large number of internal sensors and allow tuning dynamically their compliance (see III-A.1. The sensors space is extended by the addition of 8 force sensors under each foot, an inertial measurement unit located in the head and two wide-angle HD cameras. In addition, a 4" LCD screen is located on the face for visual communication (such as emotions, interaction).

In order to develop an adapted mechanical structure, we interested ourselves in how evolution solved sensorimotor task related to locomotion and in particular bipedal locomotion. As human locomotion represents one of the finest example of mastering bipedal walking, we took functional inspiration of some elements that seem relevant to improve the locomotion of humanoid robots.

This bio-inspiration is expressed on the whole structure of Poppy. On the anatomical point of view, it reproduces the human proportions as described in the literature [9] (see Fig. 3) and their sensorimotor space organization: i.e. the main degrees of freedom (actuated and passive), an inertial unit in the head and force sensors distributed underfoot.

As explained in 1 biped locomotion is a central design goal of the Poppy platform. For this purpose, the morphological optimization is mainly expressed on the locomotive system (legs and trunks) in 


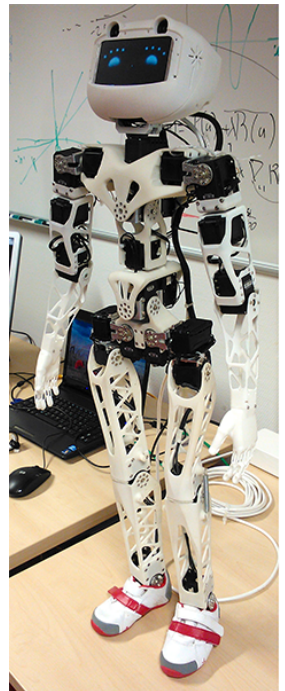

(a)

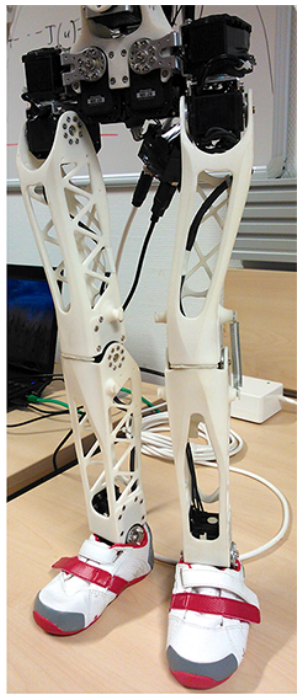

(b)
Fig. 2: a) Overview of the Poppy platform. b) Leg design.

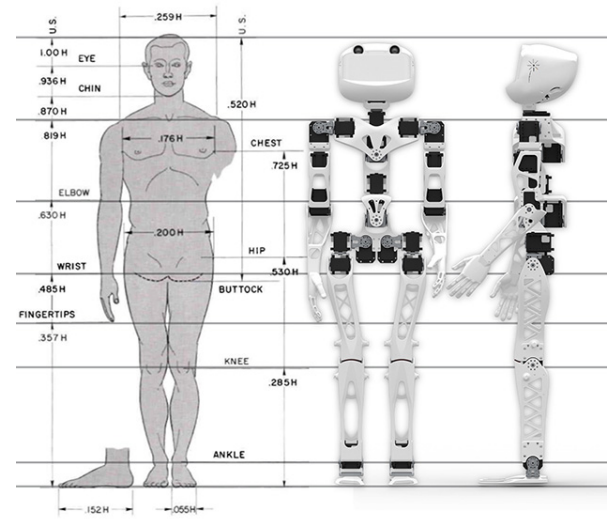

Fig. 3: Human proportion used for the design of Poppy [9]

order to increase the robot robustness, agility and stability during the walking.

\section{A. Lightweight and compliant structure}

Many humanoid robots use powerful motors often associated with highly accurate sensors. This has a cost, both in terms of weight and computation resources. Moreover, to ensure the accuracy of the sensory-motor space it is necessary to design very rigid mechanical parts. The whole structure obtained is powerful but very heavy and so not very agile. This kind of robots can intensively repeat precise and complex movements, but are somewhat uncomfortable when it comes to walking on uneven ground.

Following ecological principles [31] we decided to design a lightweight and compliant robot requiring low actuation power. All our design choices, such as the materials, the motors or the sensors, have been made in this direction and to try to tackle the challenges presented in the introduction. In the next sections we will detail each part of the robot and how they fit within these designs principles.

1) Actuation: While emerging technologies such as linear motor, artificial muscle or using both motors and cables are promising, they are still not "plug'n'play" solutions (e.g. require air circuit, associating motor and cable is a complex task, pistons are heavy and slow). It makes their integration in a small platform such as Poppy difficult.

We therefore chose to use Robotis Dynamixel servo-motor $2^{2}$ for the robot actuation. They are all-in-one-modules which contain drivers, encoders and communication lines. They are also quite powerful, robust and precise. This is done by the combination of maxon motor, metal gear box and precise position sensor (resolution: $0.1^{\circ}$ ). They embed a 32bits micro-controller dedicated to the communication (serial port), the control of the joint (position, speed or torque) and the measurement of severals internal data such as the real position, speed, load or temperature. They also allow tuning the internal PID or limiting the maximal torque. This permits rich behaviors useful both for physical interaction and locomotion. However these motors are quite heavy (72, 126 and $153 \mathrm{~g}$ respectively for MX-28, MX-64 and MX-106) in comparison of the Futaba servo-motor ${ }^{3}$ 20-50g for a comparable output torque. Given these constraints, the challenge consists in minimizing the number of motors and the power needed to reduce the global weight of the robot.

It would be interesting to mix both robotis motors with basic servo-motors where the compliance is not so important. However, as this makes the design more complex we did not explore this solution yet.

2) Material properties: All mechanicals parts are made using laser sintering technology. This 3D printing process allows the production of almost any shape without constraint. In addition, the price of the part depends on the total size and not on the complexity of the shape. This permits the production of very optimized shapes without increasing the total price of the robot. Also, this technique is compatible with several materials from polyamide to titanium. Parts manufacturing was subcontracted to an external company ${ }^{4}$

For Poppy's structure we decided to use the polyamide material because it is lightweight and very flexible while keeping good

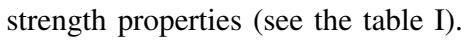

\begin{tabular}{|l||l|l|l|}
\hline Material & $\begin{array}{l}\text { Mass Density } \\
\rho\left(\mathrm{kg} / \mathrm{m}^{3}\right)\end{array}$ & $\begin{array}{l}\text { Yield strength } \\
\sigma(\mathrm{MPa})\end{array}$ & $\begin{array}{l}\text { Young Modu- } \\
\text { lus } E(G P a)\end{array}$ \\
\hline Polyamide & 930 & 49 & 1.65 \\
\hline Aluminum & 2700 & 200 & 70 \\
\hline Steel & $7500-8000$ & 350 & 200 \\
\hline Titanium & 4500 & 1200 & 114 \\
\hline
\end{tabular}

TABLE I: Comparison of material properties. The Young modulus represents the stiffness of the material while the yield strength corresponds to the maximal stress tolerable before plastic deformation.

3) Structure design: All mechanical parts were designed to optimize their weight and make the platform Poppy as light as possible. The obtained mass reduction allows the use of less powerful motors which are therefore lighter. We can thus have a lightweight robot, strong and powerful enough to perform tasks such as walking and physical interaction.

Weight reduction was achieved through the use of trellis structures. These structures, mainly used in civil engineering, are among the best technical solutions to optimize the weight/resistance ratio. All the limbs of Poppy are based on this structure and have been optimized using finite element analysis (FEA) to perform structural simulation and validate parts performance and safety factors.

The main quadratic momentum taken at the middle of the leg given the trellis structure (see Fig. 4 a) is:

2 http://www.robotis.com/xe/dynamixel_en

http://www.futaba-rc.com/servos/brushless.html 4 http://i.materialise.com/ 


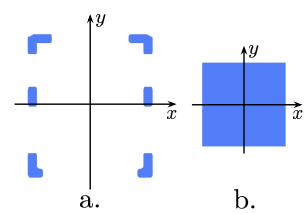

Fig. 4: a. Section of the Poppy's leg. b. Equivalent square section

$$
\begin{gathered}
I_{x}=\iint_{s} y^{2} d x d y, I_{y}=\iint_{s} x^{2} d x d y \text { with } s=d x d y \\
I_{x}=54862 \mathrm{~mm}^{4}, I_{y}=53260 \mathrm{~mm}^{4}
\end{gathered}
$$

For instance, given a solid bar with rectangular profile (Fig. 4 b):

$$
I_{x}=\frac{b \cdot h^{3}}{12}, I_{y}=\frac{b^{3} \cdot h}{12}
$$

It would require a section such as $b=27.72 \mathrm{~mm}$ and $h=$ $27.59 \mathrm{~mm}$ to get the same quadratic momentum. Considering the length of the leg part (i.e. $190 \mathrm{~mm}$ ), the total mass would be equal to $142 \mathrm{~g}$ instead of $47 \mathrm{~g}$ for the actual leg. This corresponds to a reduction of $70 \%$ of the mass.

By using this mesh structure on most of the robot, the total weight of the $3 \mathrm{D}$ printed parts were reduced of about $1.3 \mathrm{~kg}$ while still being resistant under shocks and falls.

\section{B. Feet}

To allow efficient and human-like walking gait, Poppy's feet design takes some functional inspiration from the actual human foot such as the proportion, compliance and toes which are key features concerning both the human walking [14] and biped robots with a human-like gait [35]. In addition, we wanted to reduce the weight (i.e. reducing inertia) of the feet to increase the robot agility.

To keep the foot as light as possible while conserving functional properties we decided to use a single motor for the main motion (sagittal plane). The toes articulation is done through material flexibility (see Fig. 55 while lateral motion is obtained by the shoes compliance.

This results in a foot that weights less than 200 grams (shoe: $60 \mathrm{~g}$ ) and which is small compared to conventional humanoids. However, that raises problems concerning balance because the support polygon is reduced. We thus decided to add 8 FSF pressure sensors under each foot to have accurate feedback of the current state of the robot (see Fig. 5. The number of sensors is limited by the number of analogical port on an Arduino Nano board. It would be possible to add more sensors but it would be more complicated to build.

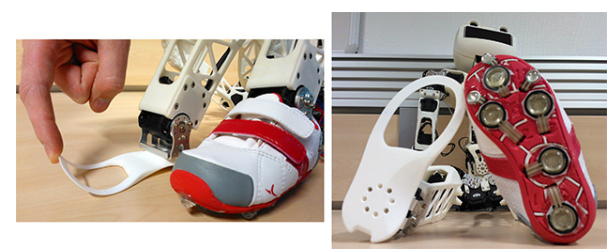

Fig. 5: Poppy's feet. left: Toes articulation is done by compliance using material properties. right: 8 FSR Sensors are placed under each foot.

\section{Hip}

As mentioned above, Poppy's small feet increase the challenge of the balance of the robot. Also, to keep the projection of the center of gravity $(\mathrm{CoG})$ inside the support polygon, defined by the feet geometry, it is necessary to control the weight distribution of the robot structure. In particular, we wanted that in its initial upright posture,
Poppy stays balanced without any control. Robotis actuators are among the densest elements in the Poppy platform $\left(1700 \mathrm{~kg} \mathrm{~m}^{3}\right)$ and are the main source of weight $(1.8 \mathrm{~kg})$. Their spatial distribution represents therefore the major part of the distribution of masses in Poppy. In order to limit the displacement of the mass on the back of the robot, we decided to avoid conventional ball joint assembly for the hip joint such that it is made on most robots based on Robotis motors (i.e. distributed in a plane parallel to the sagittal plane). Instead, we placed them on the frontal plane as the from left to right stability is greater than the from rear to front stability. By doing so, the hip joint is not a real ball joint anymore. Yet, the lost freedom is not relevant for the walking gait.

With this constraint, there are two main solutions for the motor repartition (see Fig. 6a and 6p). We chose the second one (b) for the four following reasons:

- It is more compact and closer to the human proportion.

- Hip rotations (in frontal plane) lead to slight vertical motions of the leg which act as a damper during walking. This damping can be tuned by adjusting the stiffness of the actuator.

- It reduces the hip joint lever arm and thus reduces the torque required to maintain position in single support phase.

- As shown in Fig. 6c, the structure can be shown as a four-bar linkage. Following the Grasshoff's Theorem, motion characteristics of a-four-bar mechanism will depend on the ratio of the link length dimensions. Depending on the bar dimensions, structure can easily collapse if no torque is applied on the joint when subject to horizontal forces. In our case we avoid the double crank motion (links can have a full rotation) of the case (a) by using the configuration (b) which only permits a double rocker motion (links can only oscillate).

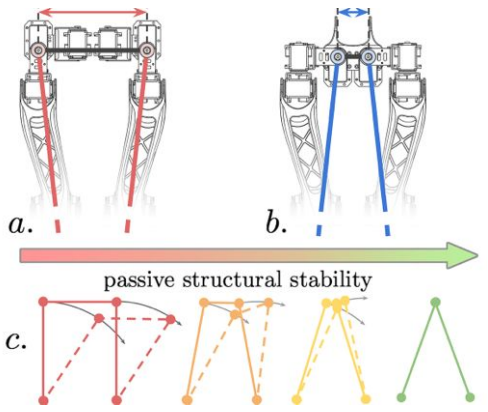

Fig. 6: The two possible choices (a) and (b) for the hip articulation of poppy. (c) Structural instability depending on the shape of the structure.

\section{Thigh}

If we look closely at the human morphology of the femur, it appears that it is inclined of 6 degrees. This makes the feet closer to the projection of the center of gravity (see Fig.7 a). We reproduced this on Poppy. While we could have inclined the whole leg, we chose to only incline the upper part of the leg to make all the motors of the leg actuate in the same plane. Both approaches lead to the same two main stability enhancements during walking gait:

1) Reducing the lateral falling speed: We can model the situation where the robot is on one foot by an inverted pendulum with a point mass centered on the CoG of the robot and the axis of rotation located at the foot position (see Fig. 7b). The dynamic of the whole structure depends on:

- the length $l$ of the segment extending from the foot to the center of gravity, 


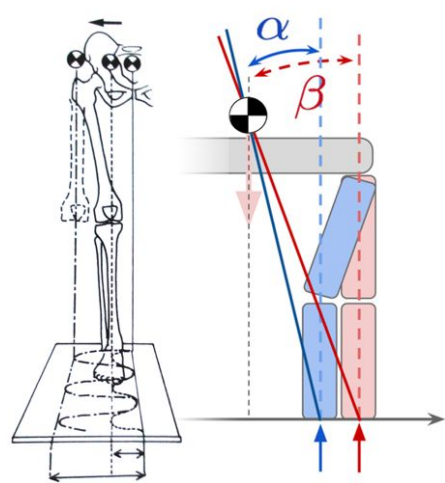

Fig. 7: left: Human leg anatomy and effect of the bending femur on the CoG motion during walking gait. right: Model for the dynamic comparison.

- the angle $\theta$ of the segment relative to the vertical,

- the gravity force $g$.

The system follows the law:

$$
\ddot{\theta}(t)+w_{0} \cdot \sin (\theta(t))=0, w_{0}=\sqrt{\frac{g}{l}}
$$

To get a first idea of the behavior, we can linearize the system for small disturbance by:

$$
\begin{gathered}
\theta(t)=\theta_{0} \cdot \cos \left(w_{0} \cdot t\right) \\
\dot{\theta}(t)=-\theta_{0} \cdot w_{0} \cdot \sin \left(w_{0} \cdot t\right)
\end{gathered}
$$

The position and velocity of the pendulum varies linearly with the initial $\theta$ angle. Reducing this initial angle involves a direct reduction of the falling speed $\dot{\theta}(t)$ of the robot.

In the case of the geometry of Poppy, the thigh bending permits a $40 \%$ reduction of the initial angle $\theta_{0}\left(\alpha=3.8^{\circ}\right.$ against $\beta=6.4^{\circ}$ on Fig. $7 \mathrm{~b}$ ).

In the case of a fall, it is not possible to respect the assumption of small perturbations, that is why we have simulated the model in Matlab with a non-linear system. We obtain the behavior represented in Fig. 8
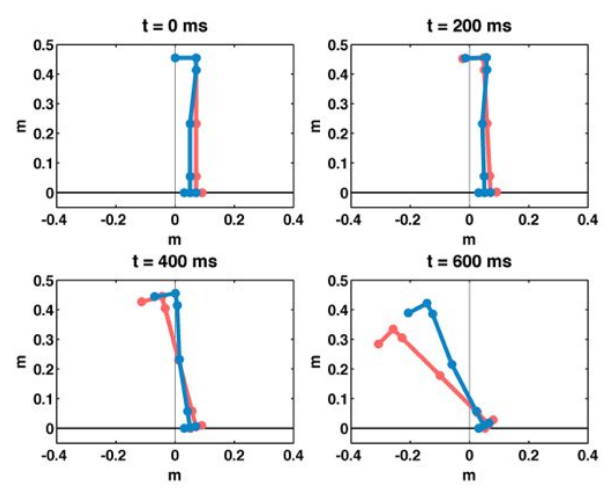

Fig. 8: Comparison of the structure's dynamic over time when Poppy is standing on one foot depending on the thigh morphology: with a bended thigh of $6^{\circ}$ (blue) and without (red).

If we define the center of gravity altitude as:

$$
z_{C o G}=l \cdot \cos (\theta(t))
$$

We can express its falling speed over time as:

$$
\dot{z}_{C o G}=-\dot{\theta}(t) \cdot l \cdot \sin (\theta(t))
$$

The simulation shows that between 0 and $700 \mathrm{~ms}$, the mean of the CoG falling speed is reduced by $60 \%$.
2) Reducing the lateral translation of the center of gravity: As the feet are closer to the gravity center, the necessary lateral translation of the center to go from one foot to another is reduced (see Fig 7 a). In the case of the Poppy morphology thanks to the $6^{\circ}$ bended thigh, the lateral motion of the CoG is reduced by about $30 \%(5 \mathrm{~cm}$ instead of $7.1 \mathrm{~cm})$.

\section{E. Semi-Passive Knee}

The Poppy platform involves a semi-passive knee based on the use of additional springs in parallel of the joint actuation. These springs have been design to participate in the leg dynamic during two main phases:

- They help to keep the leg straight during the support phase without any motor control.

- During the swing phase, they participate to the flexion of the leg.

These two modes are passively switched by the actual knee angle. Considering the human knee kinematic (see Fig. 9), we chose to change mode at $\theta_{k n e e}=20+5^{\circ}$ which corresponds to a transition between the preparing stance phase and the swing phase.

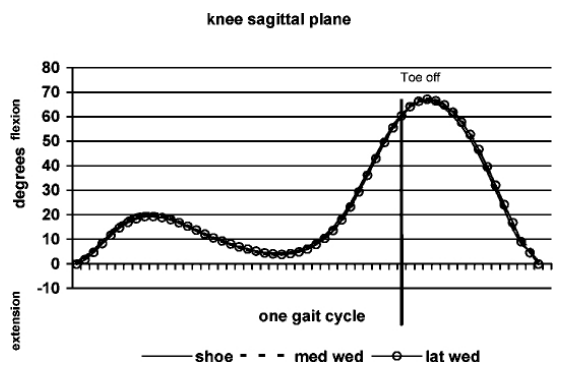

Fig. 9: Actual human knee flexion kinematic during the walking gait [23]. We can identify two main phases corresponding to the preparation of the stance phase and the swing phase. The main difference is the amplitude of the motion i.e $<20^{\circ}$ for the stance phase and $>20^{\circ}$ for the swing phase.

We performed a parametric optimization both on the position of the spring ties $\left(M_{T}\right.$ and $\left.M_{L}\right)$ and on its characteristic $\left(K, L_{0}, D_{i}\right.$, $F_{\max }, L_{\max }$ ) (see Fig. 10 to try to match the above mentioned criteria. These criteria are modeled as condition on the resultant torque:

- $C(\theta=0)<-0.4$ : Locking of the knee, where $0.4 N . m$ is the necessary torque to keep the leg straight.

- $C\left(\theta=25^{\circ}\right)=0$ : Transition between the two behaviors

- $C>0$ if $\theta>25 \mathrm{deg}$ : Helps the motor to lift the leg.

- $\max (|C(\theta)|)<\frac{C_{M X-28}}{2}$ : The actuator $M X-28$ should always be powerful enough to control the joint motion.

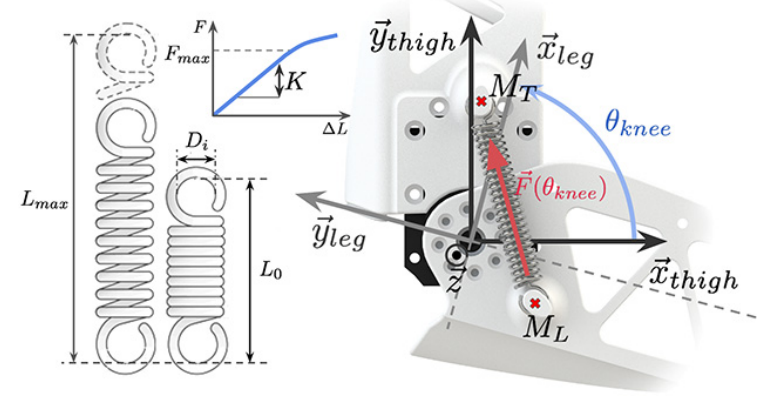

Fig. 10: Spring parameters to optimized 
The resultant torque $C$ generated by springs in function of the knee flexion $\theta$ ( $\left.n_{\text {spring }}=2\right)$ is computed as follow:

$$
\begin{aligned}
& C(\theta)=\left.n_{\text {spring }} \cdot \overrightarrow{O M_{L}}\right|_{R_{\text {thigh }}} \wedge \vec{F}(\theta) \cdot \vec{z} \\
& \text { with: } \\
& \|F(\theta)\|=K \cdot\left(L(\theta)-L_{0}\right), \quad l(\theta)=\left\|\left.\overrightarrow{M_{T} M_{L}}\right|_{R_{\text {thigh }}}\right\| \\
& \qquad\left.\overrightarrow{O M_{L}}\right|_{R_{\text {thigh }}}=\left.\overrightarrow{O M_{L}}\right|_{R_{\text {leg }}} \cdot\left[\begin{array}{ccr}
\cos (\theta) & -\sin (\theta) & 0 \\
\sin (\theta) & \cos (\theta) & 0 \\
0 & 0 & 1
\end{array}\right]
\end{aligned}
$$

We use an iterative selection on these criteria to determine the appropriate characteristics for the spring.

a) Minimizing stresses on the structure: The length of the lever arm is constrained by the dimensions of the legs, resulting in an increase of the force generated by the spring to produce the desired torque on the knee.

By maximizing the following criterion with the constraint $C(\theta=$ $\left.25^{\circ}\right)=0$ :

$$
c_{1}=\frac{C_{\max }}{F_{\max }^{2}}
$$

We were able to determine the ties specific location $\left(M_{T}\right.$ and $M_{L}$ ), for both minimizing mechanical stress and changing the torque direction for $\theta=25^{\circ}$,

$$
M_{T}=\{2,39,0\}_{\text {Rthigh }} M_{L}=\{-12,23,0\}_{\text {Rleg }}
$$

and constraints concerning the springs characteristics:

$$
L_{\min }<42.6 \mathrm{~mm} \quad L_{\max }>65.12 \mathrm{~mm}
$$

b) Ties strength: We calculated the minimum diameter of the ties so that it can withstand the constraints imposed by the spring with a beam theory model:

$$
D_{\text {min }}=\sqrt[3]{\frac{32 \times C_{s} \times F_{\text {max }} \times l_{\text {tie }}}{2 \pi \times \sigma_{\text {Max Polyamide }}}}
$$

By considering Poppy's parameters and a coefficient of safety $C_{s}=$ 5 , we found that the spring must respect the criterion $D_{\min }>$ $6.5 \mathrm{~mm}$.

Considering the desired spring behavior and geometrical conditions, an automatic selection over 720 different spring 5 was performed. Only 5 springs satisfied all criteria. For the Poppy platform we chose a spring with the following characteristics: $\left\{D_{i}=9.6 \mathrm{~mm}, L_{0}=42 \mathrm{~mm}, K=1620 \mathrm{~N} . \mathrm{m}^{-1}, F_{\max }=81.7 \mathrm{~N}\right.$, $\left.L_{\max }=72.8 \mathrm{~mm}\right\}$ inducing a resultant behavior shown in Fig. 11 As we can see, even if the torque applied by the spring is quite low $\left(C_{\max }=0.74 \mathrm{~N} . \mathrm{m}\right)$, the force subjected to spring ties is up to $40 N$. The shape of this ties has been optimized using FEA in order to handle the stress.

An illustration of the real behavior is shown in the attached video 6

\section{F. Trunk}

Poppy uses the bio-inspired trunk system introduced by Acroban [19]. Using five servo-motors, it allows the reproduction of the main DOFs of the human spine [7]. This feature permits the integration of more natural and fluid motion while improving the user experience during physical interaction. In addition, the spine plays a fundamental role in bipedal walking and postural balance by actively participating in the balancing of the robot.

Contrary to the design of the hips, it was not possible here to fit the 5 motors in the frontal plane due to the limited space in the trunk. So to reduce the shifting of the center of gravity to the back

${ }^{5}$ pre-selection of springs in the vanel.com catalogue flowers.inria.fr/IROS2013/poppy_knee.m4v

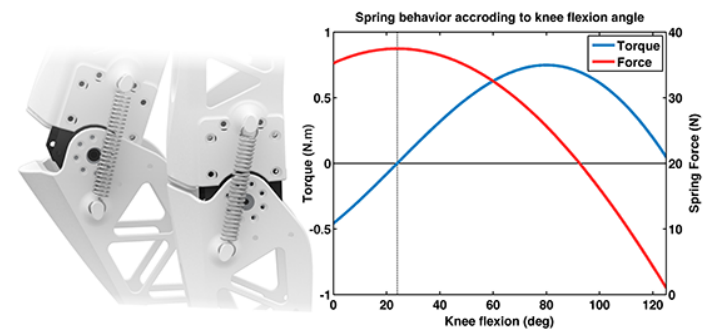

Fig. 11: Actual spring behavior for the semi-passive knee. The blue line corresponds to the torque applied by the spring on the leg according to the flexion angle of the knee. The red line corresponds to the force that the spring applied on ties.

of the robot we gradually shifted the upper body to the front. By doing so, we keep the CoG in the support polygon.

\section{G. Electronic Architecture}

The sensorimotor control of the robot is locally performed by different calculation units that outsource the low-level control. The whole is then transmitted to a computer via USB. The motor control is done via a USB2AX dongle 7 which allows a very fast sensorimotor loop for motors such as Robotis AX and MX, thanks to synchronized readings and writings on the communication bus. To ensure optimal speed, we use two USB2AX dongles (upper and lower body) that permit a parallelization of the low-level communication.

Regarding the sensors (force sensors and IMU), each unit is associated with an Arduino device which makes a local precomputation before forwarding the result to the computer. Two Arduino nano boards are placed above the ankles for the acquisition of the force underfoot and to compute the center of pressure. In the head, a Razor Sparkfun board, based on an Arduino mini, measures the acceleration values, the speed of rotation and the compass to calculate the absolute orientation of the head. It is performed by a modified version of the Razor IMU AHRS code 8

\section{H. Control software}

We use our own library called PyPot? to control robots based on robotis motors. It implements the dynamixel serial communication protocol to read/write values on the motor registers. This library has been entirely written in Python as it allows fast development, easy deployment on all operating system and quick scripting by non-necessary expert developers. It also offers a large variety of scientific and machine learning libraries used in robotics. This language is rather slow compare to $\mathrm{C}$ or Java, yet as the serial communication is handled through the standard library we can still achieve rather high performance (sensorimotor loop at $50 \mathrm{~Hz}$ ).

We performed a series of test to evaluate how fast we can communicate with robotis motors. Thanks to the USB2AX device and in particular to its SYNC_READ instruction we can read and write the position of 15 motors in about $5 \mathrm{~m}^{10}$ Reading contiguous registers (such as the position/speed/load or the pid gains) can be done in almost the same amount of time as accessing a single register. The read/write of position, speed and load takes about $6 \mathrm{~ms}$

\footnotetext{
${ }^{7}$ developed as an open-source project by Paranoid Studio

8 https://dev.qu.tu-berlin.de/projects/ sf-razor-9dof-

/https://bitbucket.org/pierrerouanet/pypot

${ }^{10}$ All tests have been made on a linux. Those results can significantly change depending on the USB2serial driver.
} 
with 15 motors. We use a very fast writing mode (about $100 \mu s$ ) that does not wait for the motor acknowledgment. It is important to note that the time of the read/write loop is linear in number of motors. On the Poppy platform we use two USB2AX controllers (one with 10 motors and the second with 15) which allow for a $6 \mathrm{~ms}$ motor loop (position/speed/load).

\section{EXPERIMENTS}

As explained in previous sections, the Poppy platform was designed for walking experiments and explore physical interaction with user. In this section we will present an initial experiment to show properties of the robot when walking with the physical guidance of a human and to explore possibilities and limitations of the current platform.

The experiment consists in playing an open-loop walking pattern while the robot is guided through the physical interaction of a human. The user role is to provide both balance and control of mass transfer. By producing small lateral motion on the upper-body he can act on the robot to move its CoG from one foot to another as illustrated in the attached vided 11

The gait is basically based on the actual human sagittal joint kinematic [23]: hip, knee, ankle (see Fig. 12 a). A direct transposition of the human joint kinematic on the Poppy's morphology results in a walking speed too fast to be handled by users (see Fig. 12 b). A simple reduction of joints amplitude conducts to an unsuitable leg trajectory where toes bump into the ground during the swing phase (see Fig. 12 c). So to ensure enough clearance during the swing phase and suitable walking speed for the guidance with user, we modified the joints trajectories by hand to both reduce the length step and increase the foot clearance (see Fig. 12 d). (a)

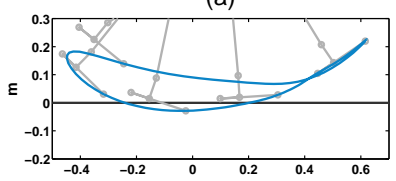

(c)

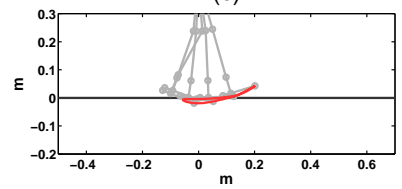

(b)

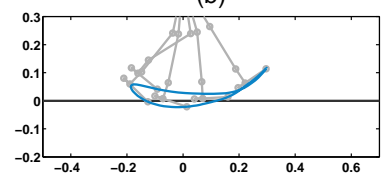

(d)

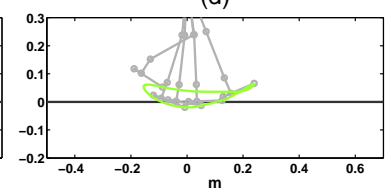

Fig. 12: Toes trajectories generated by the walking pattern. a) Kinematics of human walking with human's morphology. b) Direct transposition of the human kinematics with Poppy's morphology. c) Reducing amplitude of the human kinematics joints with Poppy's morphology. d) Walking pattern used for the experiments.

\section{RESULTS}

\section{A. Compliant Motion}

We added a controller dedicated to control the compliance of the knee depending on its current state (i.e. position-velocity). If the spring can provide assistance to the joint motion then the controller turns the actuator in full compliant mode. During walking gait, this controller enables the compliance from 35 to $45 \%$ of the gait cycle.

In addition, another controller turns compliant the ankle joint when the heel hits the ground. This provides a compliant motion for $30 \%$ of the gait cycle and participates to the robot's adaptivity to the ground.

\footnotetext{
11 flowers.inria.fr/IROS2013/poppy_walk.m4v
}

\section{B. Powered Enough}

By using stiff joints in the shoulder we were able to measure shoulder torque and evaluate ${ }^{12}$ the forces applied by users while they were helping Poppy to walk. Over 26 min of experimentations, the mean forces applied by the user were $0.75 \pm 0.15 N$ for sagittal balance and $0.3 \pm 0.03 N$ for lateral mass transfer. So the user only provides little input to the robot. Also as we can see in Fig. 13 a, the global reaction forces recorded by FSR sensors show that Poppy is supporting its own weight (i.e. $35 N$ ) during all the cycle gait. In addition, the electrical global consumption of the robot is about 30-38W during walking ${ }^{13}$ (idle 15-18W).
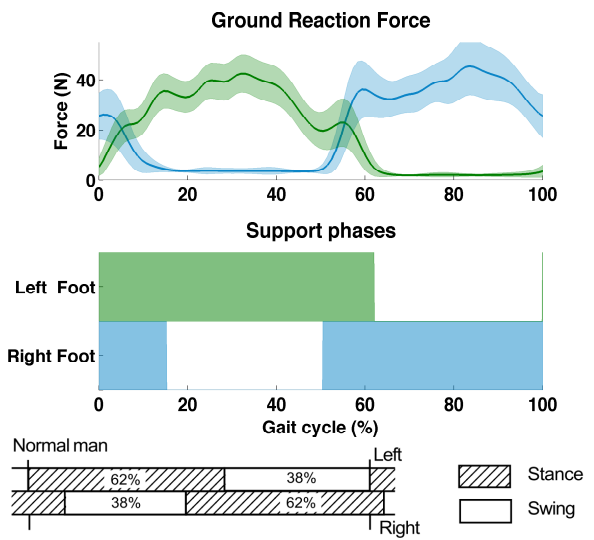

Fig. 13: top: Mean and standard deviation of ground reaction forces measured under left and right foot (resp. green and blue) during cycle gait. middle: Support phase duration for each foot computed from GRF data. bottom: Support phase duration for each foot during human walking gait [39]

\section{Human like gait}

The analysis of this experiment shows similarities between the human and Poppy's gait. Indeed, the mean stance phase duration (see Fig. 13 b) was $64.1 \%$ and $62.1 \%$ (respectively for the left and right foot ${ }^{14}$ which is close to the typical human walking gait [39] (see Fig. 13 c).

This initial experiment is very encouraging regarding Poppy's ability to reproduce a human-like walking gait with low-powered motor and low-control as we showed that the user contributions is quite weak.

\section{CONCLUSION}

In this paper we presented the design rules we followed to create in 4 months a new humanoid platform called Poppy. This platform was specifically designed to study biped locomotion and compliant physical interaction. We presented an initial experiment which shows very promising results concerning the biped locomotion. In particular, we showed that with little physical help from user, Poppy can produce a walking gait very similar to the human one. Yet, our first experiment leads us to reconsider part of Poppy's design to improve it. In particular, the trunk uses 5 motors with a range of $360^{\circ}$. This is not really useful as only small range are needed here. We could replace this by a lighter design.

\footnotetext{
${ }^{12}$ by using the arm length as lever arm

${ }^{13}$ When the robot overworks the consumption can raise up to $65 \mathrm{~W}$

${ }^{14}$ The foot fabrication is hand made which lead to asymmetric morphology and can explain this slight difference.
} 
Poppy was also conceived while considering issues of dissemination and was optimized to be very accessible (i.e. cheap and easy to assemble) as our midterm goal is to make the whole hardware and software platform available for academics. Current foot design has to be optimized to make it easily reproducible by other people (same shoe is needed and the connection of foot sensors is tricky). We are thus exploring the design of new foot with integrated lateral compliance and force sensors. Current hands are only "handle" used for physical interaction. On future work, we would like to actuate these hands in a compliant way to explore grasping tasks.

\section{REFERENCES}

[1] B. D. Argall, S. Chernova, M. Veloso, and B. Browning. A survey of robot learning from demonstration. Robotics and Autonomous Systems, 57(5):469-483, 2009.

[2] Y. Asano, H. Mizoguchi, T. Kozuki, Y. Motegi, M. Osada, J. Urata, Y. Nakanishi, K. Okada, and M. Inaba. Lower thigh design of detailed musculoskeletal humanoid "Kenshiro". 2012 IEEE/RSJ International Conference on Intelligent Robots and Systems, pages 4367-4372, October 2012

[3] A. Baranes and P.-y. Oudeyer. The Interaction of Maturational Constraints and Intrinsic Motivations in Active Motor Development. In Proceedings of ICDL-EpiRob 2011, 2011.

[4] A. Baranes and P.-y. Oudeyer. Active learning of inverse models with intrinsically motivated goal exploration in robots. Robotics and Autonomous Systems, 2012.

[5] L. Berthouze and M. Lungarella. Motor Skill Acquisition under Environmental Perturbations: On the Necessity of Alternate Freezing and Freeing Degrees of Freedom. Adaptive Behavior, 12(1):47-63, 2004.

[6] A. Billard, S. Calinon, R. Dillmann, and S. Schaal. Robot programming by demonstration. Handbook of robotics, 1, 2008.

[7] J. C. Ceccato. Le tronc, de la locomotion à la commande. $\mathrm{PhD}$ thesis, Montpellier II, 2009.

[8] S. H. Collins, M. Wisse, and A. Ruina. A three-dimensional passivedynamic walking robot with two legs and knees. The International Journal of Robotics Research, 20(7):607-615, 2001.

[9] M. Dufour and M. Pillu. Biomécanique fonctionnelle: Membres-TêteTronc. Paris: Masson, 2005.

[10] H. Geyer, A. Seyfarth, and R. Blickhan. Compliant leg behaviour explains basic dynamics of walking and running. Proceedings. Biological sciences / The Royal Society, 273(1603):2861-7, November 2006.

[11] D. Gouaillier, V. Hugel, P. Blazevic, C. Kilner, J. Monceaux, P. Lafourcade, B. Marnier, J. Serre, and B. Maisonnier. The nao humanoid: a combination of performance and affordability. CoRR, vol. $a b s / 0807.3223,2008$.

[12] I. Ha, Y. Tamura, H. Asama, J. Han, and D. W. Hong. Development of open humanoid platform DARwIn-OP. In SICE Annual Conference (SICE), 2011 Proceedings of, pages 2178-2181. IEEE, 2011.

[13] K. Hirai, M. Hirose, Y. Haikawa, and T. Takenaka. The development of Honda humanoid robot. In Robotics and Automation, 1998. Proceedings. 1998 IEEE International Conference on, volume 2, pages 1321-1326. IEEE, 1998

[14] J. Hughes, P. Clark, and L. Klenerman. The importance of the toes in walking. The Journal of bone and joint surgery. British volume, 72(2):245-51, March 1990.

[15] A. J. Ijspeert, A. Crespi, D. Ryczko, and J. M. Cabelguen. From swimming to walking with a salamander robot driven by a spinal cord model. Science, 315(5817):1416, 2007.

[16] K. Kaneko, K. Harada, F. Kanehiro, G. Miyamori, and K. Akachi. Humanoid robot HRP-3. In Intelligent Robots and Systems, 2008. IROS 2008. IEEE/RSJ International Conference on, pages 2471-2478. IEEE, 2008.

[17] M. Lapeyre, O. Ly, and P.-y. Oudeyer. Maturational constraints for motor learning in high-dimensions: the case of biped walking. In IEEERAS International Conference on Humanoid Robots (Humanoids), pages 707-714. IEEE, 2011.

[18] M. Lungarella, G. Metta, R. Pfeifer, and G. Sandini. Developmental Robotics: A Survey. Connection Science, 15(4):151-190, 2003.
[19] O. Ly, M. Lapeyre, and P.-y. Oudeyer. Bio-inspired vertebral column, compliance and semi-passive dynamics in a lightweight humanoid robot. In 2011 IEEE/RSJ International Conference on Intelligent Robots and Systems (IROS), pages 1465-1472. IEEE, 2011.

[20] K. Matsushita, M. Lungarella, C. Paul, and H. Yokoi. Locomoting with less computation but more morphology. In Robotics and Automation, 2005. ICRA 2005. Proceedings of the 2005 IEEE International Conference on, pages 2008-2013. IEEE, Ieee, 2005.

[21] T. McGeer. Passive dynamic walking. The International Journal of Robotics Research, 9(2):62-82, 1990.

[22] G. Metta, G. Sandini, D. Vernon, L. Natale, and F. Nori. The iCub humanoid robot: an open platform for research in embodied cognition. In Proceedings of the 8th workshop on performance metrics for intelligent systems, pages 50-56. ACM, 2008.

[23] C. J. Nester, M. L. van der Linden, and P. Bowker. Effect of foot orthoses on the kinematics and kinetics of normal walking gait. Gait \& posture, 17(2):180-7, April 2003.

[24] R. Niiyama, S. Nishikawa, and Y. Kuniyoshi. Athlete robot with applied human muscle activation patterns for bipedal running. In Humanoid Robots (Humanoids), 2010 10th IEEE-RAS International Conference on, pages 498-503. IEEE, 2010.

[25] P.-Y. Oudeyer. Developmental Robotics. In N. Seel, editor, Encyclopedia of the Sciences of Learning, Springer Reference Series. Springer, 2011.

[26] P.-y. Oudeyer, A. Baranes, and F. Kaplan. Intrinsically Motivated Learning of Real-World Sensorimotor Skills with Developmental Constraints. Intrinsically Motivated Learning in Natural and Artificial Systems, 2013.

[27] P.-y. Oudeyer, F. Kaplan, and V. Hafner. Intrinsic Motivation Systems for Autonomous Mental Development. IEEE Transactions on Evolutionary Computation, 11(2):pp. 265--286, 2007.

[28] P.-Y. Oudeyer, O. Ly, and P. Rouanet. Exploring robust, intuitive and emergent physical human-robot interaction with the humanoid robot Acroban. 2011 11th IEEE-RAS International Conference on Humanoid Robots, pages 120-127, October 2011.

[29] R. Pfeifer and J. C. Bongard. How the Body Shapes the Way We Think: A New View of Intelligence (Bradford Books). The MIT Press, 2006.

[30] R. Pfeifer and F. Iida. Morphological computation: Connecting body, brain and environment. Japanese Scientific Monthly, 58(2):48-54, 2005.

[31] R. Pfeifer, F. Iida, and J. Bongard. New robotics: Design principles for intelligent systems. Artificial Life, 11(1-2):99-120, 2005.

[32] R. Pfeifer, M. Lungarella, and F. Iida. Self-organization, embodiment, and biologically inspired robotics. Science, 318(5853):1088-1093, November 2007.

[33] K. Radkhah, C. Maufroy, M. Maus, D. Scholz, A. Seyfarth, and O. Von Stryk. Concept and design of the biobiped 1 robot for human-like walking and running. International Journal of Humanoid Robotics, 8(03):439-458, 2011

[34] M. Schwarz, M. Schreiber, S. Schueller, M. Missura, and S. Behnke. NimbRo-OP Humanoid TeenSize Open Platform. In Proceedings of 7th Workshop on Humanoid Soccer Robots. IEEE-RAS International Conference on Humanoid Robots, 2012.

[35] R. Sellaouti, O. Stasse, S. Kajita, K. Yokoi, and A. Kheddar. Faster and Smoother Walking of Humanoid HRP-2 with Passive Toe Joints. 2006 IEEE/RSJ International Conference on Intelligent Robots and Systems, pages 4909-4914, October 2006.

[36] S. Steingrube, M. Timme, F. Wörgötter, and P. Manoonpong. Selforganized adaptation of a simple neural circuit enables complex robot behaviour. Nature Physics, 6(3):224-230, 2010.

[37] A. Tapus, M. J. Mataric, and B. Scassellati. Socially assistive robotics. IEEE Robotics and Automation Magazine, 14(1):35, 2007.

[38] R. Tedrake, T. Zhang, and H. Seung. Actuating a simple 3D passive dynamic walker. IEEE International Conference on Robotics and Automation, 2004. Proceedings. ICRA '04. 2004, pages 4656-4661 Vol.5, 2004

[39] C. L. Vaughan, B. L. Davis, L. Christopher, and J. C. O. Connor Dynamics of human gait, volume 26. Human Kinetics Publishers Champaign, Illinois, 2005

[40] J. Weng, J. McClelland, A. Pentland, O. Sporns, I. Stockman, M. Sur, and E. Thelen. Autonomous mental development by robots and animals. Science, 291(599-600), 2001. 